\title{
A one-pot in-situ synthesis of copper cluster doped hydrogen substituted graphdiyne nanofibers (Cu-HsGDY)
}

\author{
Wenjun $\mathrm{Hao}^{1,{ }^{*}}$, Lei $\mathrm{Jin}^{1}$, Rong Fan ${ }^{1}$, Xinyu $\mathrm{Su}^{1}$, and Zongping Chen ${ }^{1}$ \\ ${ }^{1}$ State Key Laboratory of Silicon Materials, School of Materials Science and Engineering, Zhejiang University, Hangzhou, China
}

\begin{abstract}
Graphdiyne (GDY) is a new type of two-dimensional (2D) carbon materials, in which two benzene rings are chained by diacetylenic linkages $(-\mathrm{C} \equiv \mathrm{C}-\mathrm{C} \equiv \mathrm{C}-)) \gamma-\mathrm{GDY}$ is the most studied GDY due to its stable configuration and was experimentally obtained in 2010 through cross coupling reaction by using hexaethynylbenzene as precursor. Hydrogen substituted graphdiyne (HsGDY) was obtained using 1,3,5triethynylbenzene as precursor in a similar process. Hereinto, a copper cluster doped hydrogen substituted graphdiyne nanofibers (Cu-HsGDY) were prepared through a facile one-pot in-situ synthetic approach in a good reproductive manner. Through simply removing the copper foil, the obtained robust Cu-HsGDY can be transferred onto arbitrary substrates, especially flexible substrates, such as polyethylene terephthalate (PET), which can be used as flexible electronics as future materials.
\end{abstract}

\section{Introduction}

In the great technological change at the turn of the 21st century, our daily lives have been boosted by the fast development of novel electronic instruments. Thereinto, flexible and wearable electronic devices and energy storage systems are attracting broad research interests due to their promising use in wearable human health and activity monitoring systems [1]. Non-natural carbon allotropes, including fullerene, carbon nanotubes, graphene, graphyne, and their derivatives, have contributed to various possibilities in the field of energy [2, 3], catalysis [4], environmental [5], and biomedical domains [6], due to their specific chemical, electronical, optical and mechanical properties. In 1987, graphyne was first predicted theoretically by Baughman et al. [7] It is a new type of two-dimensional carbon allotrope which contains $s p-s p^{2}$ hybridized carbon atoms. In 1997, Graphdiyne (GDY) was predicated theoretically by Haley [8] and it is the most investigated graphyne in which two benzene rings are chained by diacetylenic linkages $(-\mathrm{C} \equiv \mathrm{C}$ $\mathrm{C} \equiv \mathrm{C}$-), including $\alpha$-GDY, $\beta$-GDY, $\gamma$-GDY [9]. $\gamma$-GDY is the most studied GDY due to its stable configuration [10]. In 2010, $\gamma$-GDY was experimentally obtained in Li's group [11] through cross coupling by using hexaethynylbenzene (HEB) and copper foil as precursor and substrate, respectively. Then various modified methods have been achieved to prepare GDY with different morphologies, such as nanotubes [12], nanowires [13], and nanowalls $[14,15]$. In 2017, an interfacial strategy was reported for the synthesis of few layers of crystalline GDY through Englinton coupling reaction [16]. In 2018, Gao et al. and co-workers reported a facile van der Waals epitaxial growth method in solution using graphene as substrates [17]. Moreover, chemical vapor deposition (CVD) technique was used to fabricate GDY at $150{ }^{\circ} \mathrm{C}$ onto the surface of silver foil [18]. GDY films were also prepared using $\mathrm{ZnO}$ nanorod arrays as substrate through a self-catalysed vapor-liquid-solid method [19]. Bulk GDY powders were generated by using copper powder as catalysts [20]. Unlike HEB for the fabrication of ultra-thin GDY films, 1,3,5-triethynylbenzene (TEB) was mainly used in the fabrication of hydrogen substituted graphdiyne nanofibers (HsGDY) with three-dimensional morphologies due to its unique chemical structure. Freestanding hydrogen substituted graphdiyne (HsGDY) frameworks were achieved and applied in flexible energy storage systems [21, 22].

Copper nanoparticles have been doped into nanofibers for future materials. Polyurethane nanofibers containing copper nanoparticles was prepared and possessed good bactericidal effect [23]. $\mathrm{Cu}$ nanoparticle modified graphene sheets electrode was reported and applied as a novel non-enzymatic glucose sensor [24]. An ultralong electron copper-carbon nanotube composite fibre was synthesized and used as transparent conductive electrodes with high operational stability [25].

In this work, we fabricate copper cluster doped hydrogen substituted graphdiyne (Cu-HsGDY) nanofibers through in-situ formation of copper clusters anchored onto HsGDY nanofibers. The present approach allows growth of $\mathrm{Cu}-\mathrm{HsGDY}$ in a robust manner. The obtained $\mathrm{Cu}$ HsGDY nanofibers can be applied in flexible electronics which will be reported elsewhere.

\footnotetext{
* Corresponding author: wenjunhao@zju.edu.cn 


\section{Experimental method}

\subsection{Materials}

All the reagents were purchased from Sinopharm and used as received. Copper foil (thickness $0.025 \mathrm{~mm}, 99.8 \%$ ) was purchased from Alfa Aesar. After cleaning consecutively by ultrapure water, ethanol, and acetone for several times, the copper foil was immersed into $1 \mathrm{M} \mathrm{HCl}$ aqueous solution for $10 \mathrm{~min}$ and rinsed by ultrapure water for three times. The cleaned copper foil was dried under a flow of argon and used for the reaction immediately.

\subsection{Synthesis of Cu-HsGDY on Cu foil}

Typically, 1,3,5-triethynylbenzene (TEB, 4 mg, 0.0266 mmol) was added into a glass bottle containing $12 \mathrm{~mL}$ pyridine as solvent. The copper foil was immediately submerged into the solution after cleaning. The glass bottle was sealed with Teflon cap and heated to $60{ }^{\circ} \mathrm{C}$ in an oven for $24 \mathrm{~h}$. Repeated experiments show no significant differences regarding to the obtained $\mathrm{Cu}$ HsGDY layer morphologies and thickness. After reaction, the samples were rinsed immediately with pyridine, NMethyl pyrrolidone (NMP), and acetone sequentially. Finally, the $\mathrm{Cu}-\mathrm{HsGDY}$ on copper foil was dried by a flow of argon and golden yellow films was obtained on the copper foil.

\subsection{Transfer of Cu-HsGDY}

The copper foil was removed by ammonium persulfate aqueous solution $\left(0.25 \mathrm{~g} \mathrm{~mL}^{-1}\right)$ for several hours until the copper foil disappeared. The $\mathrm{Cu}-\mathrm{HsGDY}$ films were cleaned by ultra-pure water for several times and transferred onto arbitrary substrate. The $\mathrm{Cu}-\mathrm{HsGDY}$ films were naturally dried under ambient condition for $5 \mathrm{~h}$ and kept in vacuum overnight.

\subsection{Characterizations}

The obtained $\mathrm{Cu}-\mathrm{HsGDY}$ samples were characterized by scanning electron microscope (SEM) using a Hitachi SU8010 microscope at $5 \mathrm{kV}$. Cu-HsGDY films were also characterized by transmission electron microscope (TEM) and high resolution TEM (HRTEM) using JEOL $2100 \mathrm{~F}$ microscope operating at $200 \mathrm{kV}$. The $\mathrm{Cu}$-HsGDY were deposited on TEM grids from alcohol dispersion after a sonication step of $\mathrm{Cu}-\mathrm{HsGDY}$ on copper foil for $2 \mathrm{~min}$. Raman spectra were collected by WITec alpha300R. The surficial chemical state and composition of the assynthesized samples were characterized by XPS using a Kratos AXIS Supra spectrometer. The raw XPS data were corrected using the binding energy of the $\mathrm{C}-\mathrm{C}$ bond at $284.6 \mathrm{eV}$ and fitted with Gaussian-Lorentzian curves.

\section{Results and discussions}

It is well-known that $\mathrm{Cu}^{+}$and $\mathrm{Cu}^{2+}$ salts have been used as catalysts for cross-coupling reactions [26, 27]. Copper foil is used to generate $\mathrm{Cu}^{+}$or $\mathrm{Cu}^{2+}$ ions in alkaline solutions $[28,29]$. Thus, copper foil is used as substrate and catalyst as illustrated in figure 1a. Based on the structure of $\gamma$-GDY, large $\pi$-conjugation HsGDY would be obtained possessing larger pores and abundant $\mathrm{H}$ group in the pores compared to graphene and graphdiyne when TEB was used as the initial monomer. $\mathrm{Cu}-\mathrm{HsGDY}$ was prepared through an insitu Glaser coupling reaction in pyridine in a sealed glass vial reactor operated under ambient condition. The cleaned copper foil was pre-treated by $\mathrm{HCl}(1 \mathrm{M})$ for 10 min and immersed immediately into TEB solution in pyridine. The reactor was sealed well with Teflon cap and kept in an oven for $24 \mathrm{~h}$ at $60^{\circ} \mathrm{C}$. It was observed that the solution colour changed from colourless to pale-yellow transparent solution. After rinsed by organic solvents, matte golden yellow $\mathrm{Cu}-\mathrm{HsGDY}$ nanofibers is obtained on the copper foil surface (figure 1b).

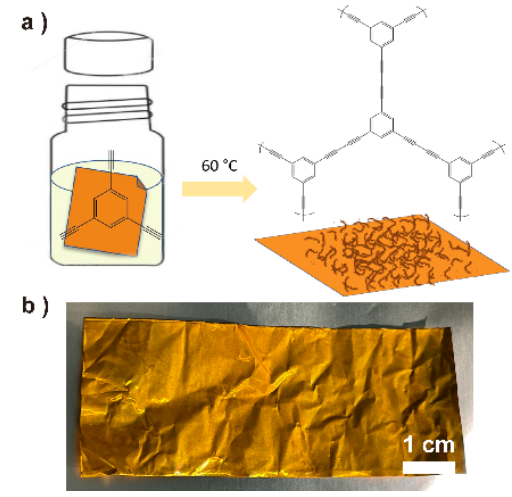

Fig. 1. a) Scheme of the synthesis process; b) Digital photograph of the obtained $\mathrm{Cu}-\mathrm{HsGDY}$ nanofibers on copper foil.

The scanning electron microscope (SEM) image in figure $2 \mathrm{a}$ is obtained from cross sectional study of the $\mathrm{Cu}$ HsGDY nanofibers removed from copper foil, which reveals that the thickness of the free-standing sample is around $1.5 \mu \mathrm{m}$. The morphology of the free-standing film close to the copper side is similar to the copper foil surface that is flat and dense. Particularly, the other side is rough and porous. This phenomenon confirms that the copper foil is served both as a substrate and as a catalyst in the synthetic process in accordance with the literature [11, 15, 21]. The surface morphologies of the obtained $\mathrm{Cu}-\mathrm{HsGDY}$ nanofibers is shown in figure $2 \mathrm{~b}, \mathrm{c}$. As illustrated in figure $2 \mathrm{~b}$, the uniformly distributed $\mathrm{Cu}-\mathrm{HsGDY}$ nanofibers possess 3D porous structures formed by bundles of nanofibers over the copper foil. As shown in figure 2c, the diameter of the formed bundles could be ranged from 5$60 \mathrm{~nm}$. 


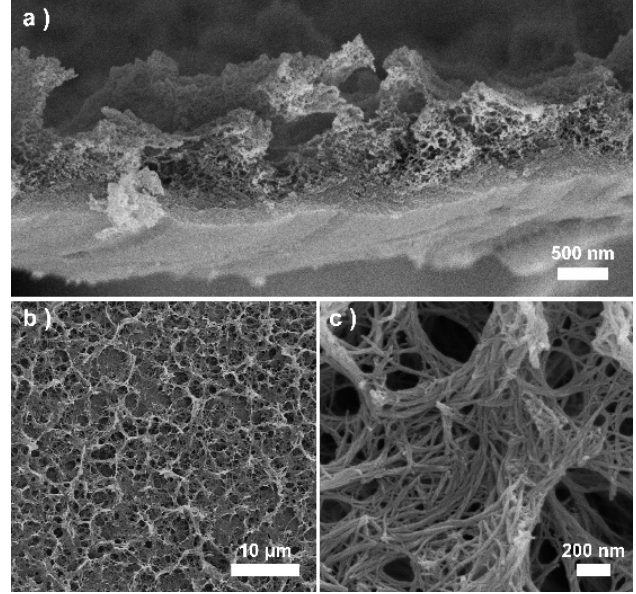

Fig. 2. a) Cross-sectional Scanning Electron Microscope (SEM) image of the free-standing $\mathrm{Cu}-\mathrm{HsGDY}$ nanofibers removed from copper foil; b,c) SEM images of the free-standing $\mathrm{Cu}$ HsGDY nanofibers on the copper foil.

The $\mathrm{Cu}-\mathrm{HsGDY}$ nanofibers on $\mathrm{Cu}$ foil were sonicated directly in ethanol solvent and distributed onto molybdenum grids. The low magnification transmission electron microscope (TEM) image (figure 3a) depicts the same bundles of nanofiber morphologies as SEM images. As depicted in figure $3 \mathrm{~b}$, the diameter of the nanofibers ranges from 3-20 nm. The High-angle Annular Dark Field Scanning TEM (HAAD-STEM) image in figure 3c illustrates that the $\mathrm{Cu}-\mathrm{HsGDY}$ nanofibers is formed by nanofibers anchored with clusters. The diameter of individual clusters ranging 1-7 nm (figure 3d) measured from high-magnification TEM (HRTEM) and HAADSTEM images (figure 3c,e). The HRTEM image (figure $3 \mathrm{e})$ depicts that the nanofiber is formed by amorphous HsGDY anchored with copper clusters. The lattice distance of the clusters is measured as $0.18 \mathrm{~nm}$ and 2.08 $\mathrm{nm}$, which correspond to $\mathrm{Cu}(200)$ and $\mathrm{Cu}(111)$, respectively. Its corresponding selected area fast Fourier transform (FFT) (figure 3f) exhibits two types of diffraction pattern which are attributed to $\mathrm{Cu}(200)$ and $\mathrm{Cu}(111)$. This observation of $\mathrm{Cu}(0)$ nanoparticles may owing to a conversion of $\mathrm{Cu}$ species to $\mathrm{Cu}(0)$ [28] or the deposition of $\mathrm{Cu}(0)$ as reported by Percec [30]. Good repeatability is achieved by characterizing the $\mathrm{Cu}-\mathrm{HsGDY}$ nanofibers obtained from different synthetic batches.

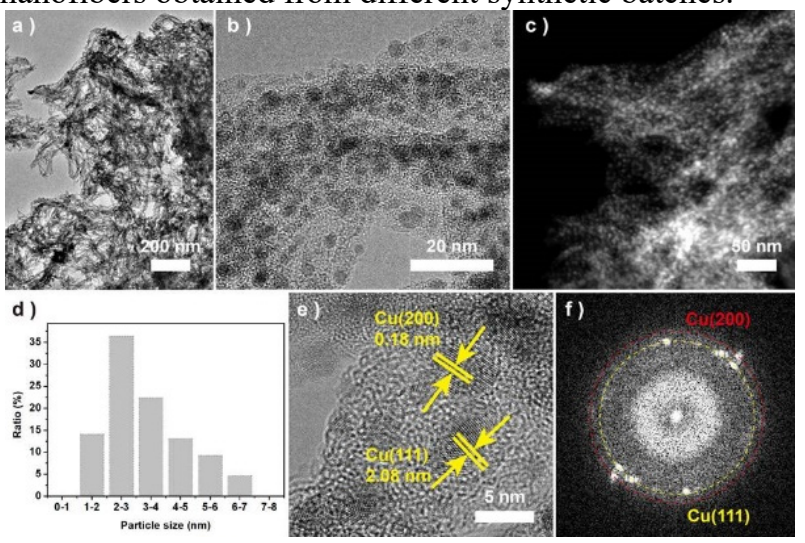

Fig. 3. a,b) Low magnification Transmission Electron Microscope (TEM) images of Cu-HsGDY nanofibers after removed away from the copper foil by sonication and c) the corresponding HAAD-STEM image; d) Histogram of the $\mathrm{Cu}$ particle size from the $\mathrm{Cu}-\mathrm{HsGDY}$ nanofibers; e) High magnification TEM image of Cu-HsGDY nanofibers and f) its corresponding fast Fourier transform (FFT) pattern.

Raman spectroscopy is a powerful technique to analyse the quality and uniformity of carbon materials. As shown in figure 4, the Raman spectrum of Cu-HsGDY nanofibers exhibits two bands centred at $996.1 \mathrm{~cm}^{-1}$ and $1590 \mathrm{~cm}^{-1}$ which are connected to the breathing and stretching in aromatic rings [31]. A strong band centred at $2218.8 \mathrm{~cm}^{-1}$ is derived from the vibration of the conjugated diyne linkage $(-\mathrm{C} \equiv \mathrm{C}-\mathrm{C} \equiv \mathrm{C}-)[11,32]$.

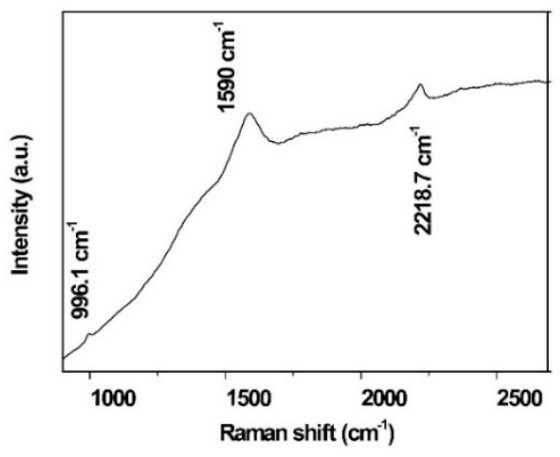

Fig. 4. Raman spectrum of the obtained Cu-HsGDY nanofibers.

X-ray photo electron spectroscopy (XPS) is used to determine the chemical composition of the Cu-HsGDY. The XPS survey scan spectrum is dominated by strong signal from carbon as illustrated in figure 5a. XPS quantitative analysis reveals the atomic ratio of different compositions as $94.79 \%(\mathrm{C}), 4.38 \%(\mathrm{O})$, and $0.83 \%(\mathrm{Cu})$. The presence of $\mathrm{O}$ impurity may due to the contamination from air. Narrow scan of $\mathrm{C} 1 \mathrm{~s}$ exhibits that the $\mathrm{Cu}-\mathrm{HsGDY}$ has both $s p$ and $s p^{2}$ hybrid carbon. The area ratio of $s p$ and $s p^{2}$ is 0.95 which is close to the chemical composition of HsGDY (figure 5b). The presence of $\mathrm{C}-\mathrm{O}$ and $\mathrm{C}=\mathrm{O}$ contribution is ascribed to sample handling under ambient condition. $\mathrm{Cu} 2 \mathrm{p}$ (figure $5 \mathrm{c}$ ) and $\mathrm{Cu}$ LMM (figure 5d) indicate that the existence of $\mathrm{Cu}(0)$ without $\mathrm{Cu}^{+}$and $\mathrm{Cu}^{2+}$ species which is in agreement with the HRTEM image and FFT pattern.
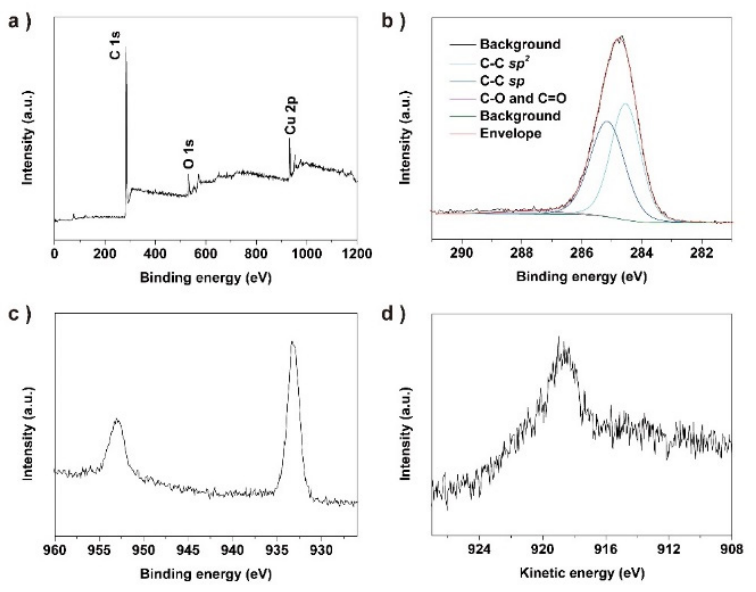

Fig. 5. a) XPS survey spectrum recorded from the $\mathrm{Cu}-\mathrm{HsGDY}$ nanofibers and its corresponding XPS high resolution scans with deconvolution across b) $\mathrm{C} \mathrm{1s}$; c) $\mathrm{Cu} \mathrm{2p}$; d) $\mathrm{Cu}$ LMM. Good agreement is observed between raw and fitting data. 
The $\mathrm{Cu}-\mathrm{HsGDY}$ nanofiber film was transferred onto a transparent polyethylene terephthalate (PET) substrate. The digital photograph (figure 6) exhibits that it is possible to bend the transferred $\mathrm{Cu}-\mathrm{HsGDY}$ on PET at any degree, which indicates that it is potential for the flexible instruments. This bendable property makes it promising in flexible electronic systems which will be report elsewhere.

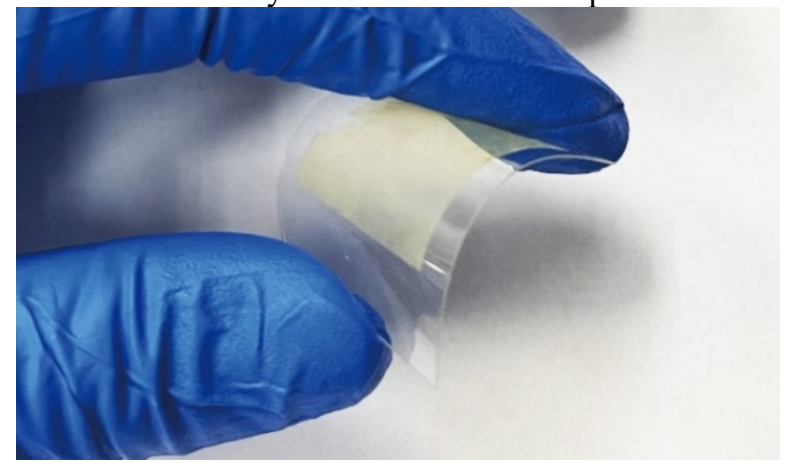

Fig. 6. Digital image of the transferred $\mathrm{Cu}-\mathrm{HsGDY}$ nanofibers onto PET substrate.

\section{Conclusions}

In summary, copper cluster doped hydrogen substituted graphdiyne ( $\mathrm{Cu}-\mathrm{HsGDY}$ ) nanofibers were prepared by a facile one-pot in-situ synthetic approach. SEM and TEM techniques reveal that the present approach allows robust and controllable growth of Cu-HsGDY. XPS and Raman spectrum indicated characteristic compliance with the chemical composition of $\mathrm{Cu}-\mathrm{HsGDY}$ with good quality. The obtained $\mathrm{Cu}-\mathrm{HsGDY}$ nanofibers can be applied in flexible electronics which will be reported elsewhere.

\section{Acknowledgments}

The authors thank the support from National Science Foundation of China (Nos. 52072336, 51902285).

\section{References}

1. Wang, C. et al. Advanced materials 31, 1801072 (2019)

2. Zhai, Y. et al. Advanced materials 23, 4828-4850 (2011)

3. Titirici, M.-M. et al. Chemical Society Reviews 44, 250-290 (2015)

4. Figueiredo, J. L. Nanotechnology in Catalysis: Applications in the Chemical Industry, Energy Development, and Environment Protection 37-56 (2017)

5. Zhang, X., Gao, B., Creamer, A. E., Cao, C. \& Li, Y. Journal of hazardous materials 338, 102-123 (2017)

6. Bardhan, N. M. Journal of Materials Research 32, 107-127 (2017)

7. Baughman, R. H., Eckhardt, H. \& Kertesz, M. The Journal of chemical physics 87, 6687-6699 (1987)

8. Haley, M. M., Brand, S. C. \& Pak, J. J. Angewandte Chemie International Edition in English 36, 836-838
(1997)

9. Malko, D., Neiss, C., Vines, F. \& Görling, A. Physical review letters 108, 086804 (2012)

10. Song, B. et al. Journal of Hazardous Materials 122957 (2020)

11. Li, G. et al. Chemical Communications 46, 32563258 (2010)

12. Li, G. et al. The Journal of Physical Chemistry C 115, 2611-2615 (2011)

13. Xue, Z. et al. ACS applied materials \& interfaces 8 , 21563-21569 (2016)

14. Gao, X. et al. Advanced Materials 29, 1605308 (2017)

15. Zhou, J. et al. Journal of the American Chemical Society 137, 7596-7599 (2015)

16. Matsuoka, R. et al. Journal of the American Chemical Society 139, 3145-3152 (2017)

17. Gao, X. et al. Science advances 4, eaat6378 (2018)

18. Liu, R. et al. Advanced Materials 29, 1604665 (2017)

19. Qian, X. et al. Scientific reports 5, 1-7 (2015)

20. Zhang, S., Liu, H., Huang, C., Cui, G. \& Li, Y. Chemical Communications 51, 1834-1837 (2015)

21. He, J. et al. Nature communications 8, 1-11 (2017)

22. Zhang, T. et al. Nature communications 9, 1-11 (2018)

23. Sheikh, F. A., Kanjwal, M. A., Saran, S., Chung, W.J. \& Kim, H. Applied Surface Science 257, 30203026 (2011)

24. Luo, J., Jiang, S., Zhang, H., Jiang, J. \& Liu, X. Analytica chimica acta 709, 47-53 (2012)

25. Daneshvar, F. et al. ACS Applied Electronic Materials 2, 2692-2698 (2020)

26. Siemsen, P., Livingston, R. C. \& Diederich, F. Angewandte Chemie International Edition 39, 26322657 (2000)

27. Li, Y.-N., Wang, J.-L. \& He, L.-N. Tetrahedron letters 52, 3485-3488 (2011)

28. Zhang, T., Du, Y., Müller, F., Amin, I. \& Jordan, R. Polymer Chemistry 6, 2726-2733 (2015)

29. Zhang, T. et al. Polymer Chemistry 6, 8176-8183 (2015)

30. Nguyen, N. H., Sun, H.-J., Levere, M. E., Fleischmann, S. \& Percec, V. Polymer Chemistry 4, 1328-1332 (2013)

31. Zhang, X., Zhou, Q., Huang, Y., Li, Z. \& Zhang, Z. Sensors 11, 11510-11515 (2011)

32. Haley, M. M. Pure and Applied Chemistry 80, 519532 (2008) 